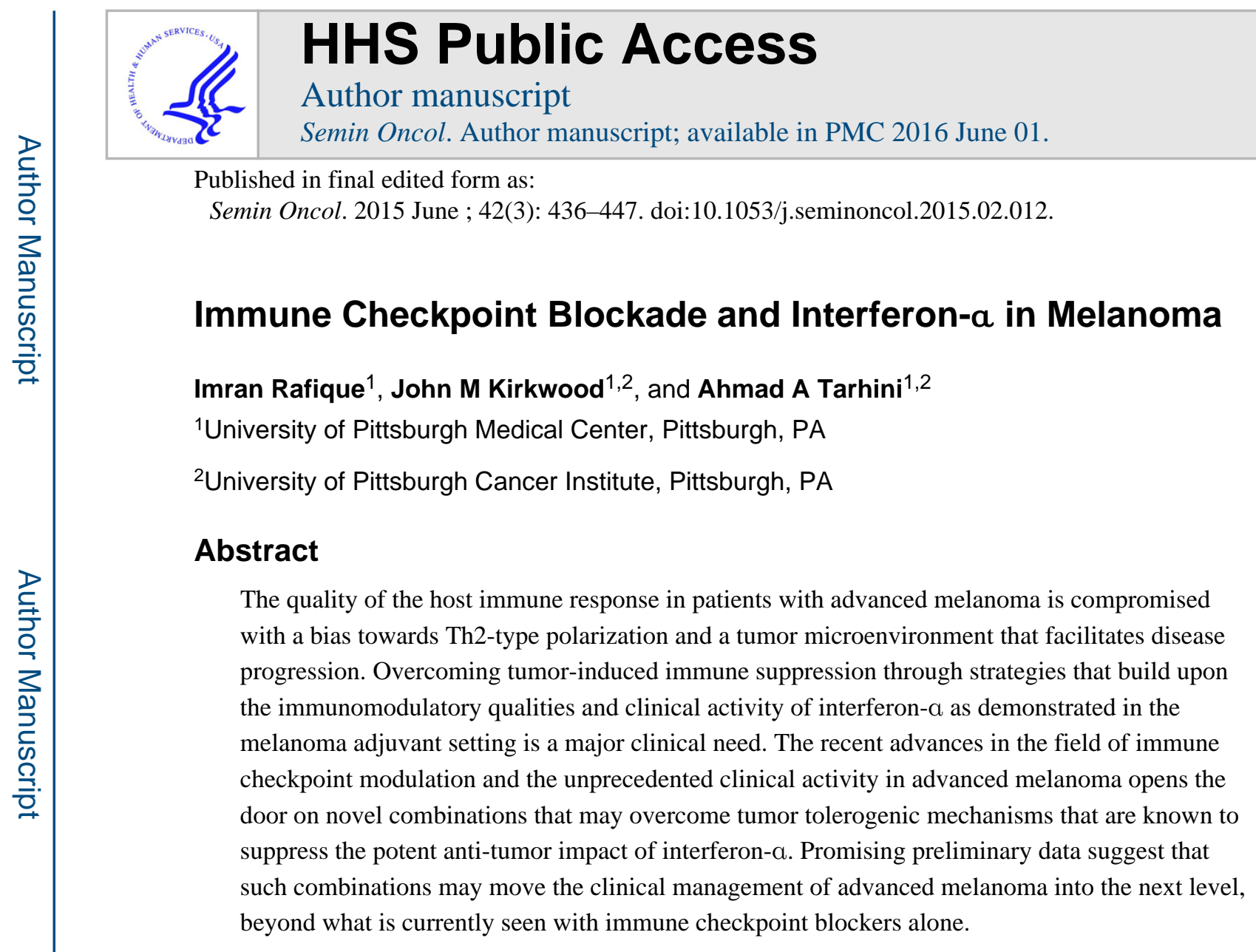

\title{
Introduction to melanoma
}

Melanoma is now the fifth and seventh most common cancer among men and women, respectively, in the United States. It is estimated that 76,100 men and women will be develop melanoma in 2014 and that of these 9710 will die.[1] According to Surveillance, Epidemiology and End Results (SEER) data the incidence of melanoma continues to rise among whites with a $60 \%$ increase observed within the last 30 years [2]. Worldwide, the incidence melanoma is also significant. Australia has the largest incidence of melanoma with rates as high as twice that of the United States.

\section{Interferon-alpha (IFN-a)}

Interferons (IFN) are a group of naturally existing glycoproteins that are secreted by cells in response to viral infections as well as synthetic and biologic inducers. Since the discovery of IFNs more than 50 years ago, in vitro and in vivo assays have demonstrated a diverse spectrum of biological activity, including antiviral, antiproliferative, and immunomodulatory properties [3]. There are different types of interferons. Type I interferons include IFN-a,

\footnotetext{
(C) 2015 Published by Elsevier Inc.

Corresponding Author: Ahmad A. Tarhini, MD, PhD, University of Pittsburgh Cancer Institute, 5150 Centre Avenue (555), Pittsburgh, PA 15232, Phone: 412-648-6578, FAX: 412-648-6579, tarhiniaa@upmc.edu.

Publisher's Disclaimer: This is a PDF file of an unedited manuscript that has been accepted for publication. As a service to our customers we are providing this early version of the manuscript. The manuscript will undergo copyediting, typesetting, and review of the resulting proof before it is published in its final citable form. Please note that during the production process errors may be discovered which could affect the content, and all legal disclaimers that apply to the journal pertain.
} 
IFN- $\beta$, IFN- $\varepsilon$, IFN- $\kappa$, and IFN- $\omega$. Type II interferons include IFN- $\gamma$, and type III interferons include IFN- $\lambda$.

Type I interferons (IFN- $\alpha$, IFN- $\beta$ ) bind to cell surface receptors with two distinct subunits: IFN-a receptor 1 and IFN-a receptor 2 . This binding triggers phosphorylation of janus kinase 1 (JAK1) and tyrosine kinase 2 (TK2), members of the Janus kinase family of receptor-associated tyrosine kinases. These kinases proceed to phosphorylate signal transducers and activators of transcriptions 1 and 2 (STAT1 and STAT2), which belong to a group of latent cytoplasmic transcription factors. The activated STAT1 and STAT2 proteins complex with p48 protein to form the IFN-stimulated gene factor 3 (ISGF3) transcription factor. ISGF3 translocates to the nucleus, where it binds to IFN-stimulated response elements in the promoters of type I IFN-responsive genes and thereby activates transcription IF'N-a's mechanism of action in melanoma is thought to be immunomodulatory rather than cytotoxic or anti-angiogenic. IFN- $\gamma$ signals through the cell surface receptor IFNGR, which consists of IFNGR1 and IFNGR2 chains, impacting distinct but related pathways to those of type I IFN. IFN- $\lambda$ signals through the unique receptors IFNLR1 and IFN-10R2 [4-7].

Among the IFNs, IFN-a2 has been the most broadly evaluated clinically. Three commercially available subspecies exist including IFN-a2a (Roferon-A, Roche Pharmaceuticals), IFN-a2b (Intron A, Merck), and IFN-a2c (Boehringer/Ingelheim). At the molecular level, IFNa has multiple effects in a variety of malignancies that range from antiangiogenic to potent immunoregulatory, differentiation-inducing, antiproliferative, and proapoptotic effects [8]. It has significant effects in relation to promoting tumor immunogenicity and enhancing dendritic cell (DC) response to tumor, DC polarization or maturation, survival and antigen cross-presentation that leads to anti-tumor immunity [810]. IFN-a promotes a shift in host immunity against tumors from Th2 bias toward Th1, enhancing cell-mediated cytotoxicity and has a role in attracting Th1 lymphocyte traffic to the tumor [11-18]. Recently, type I IFNs have been found to be critical to the innate immune recognition of a growing tumor in vivo leading to intratumor accumulation of CD8a+ DCs that promote tumor antigen-specific CD8+ T cell responses in the mouse.[19]

\section{Adjuvant IFN-a for high-risk resected melanoma;}

Randomized clinical trials over the past 30 years have established high dose IFN-a (HDI) as the mainstay of adjuvant therapy for the treatment of resected stage IIB/III melanoma. More recently, pegylated IFNa achieved regulatory approval in the U.S. as adjuvant therapy for stage III melanoma.

IFN- $a$ regimens that have been tested in randomized adjuvant trials may be classified according to the dosages employed into low-dose, intermediate-dose and high-dose categories. These regimens also vary in terms of the duration of therapy, route of administration and the type of IFN used. Patients targeted in these trials may be categorized according to the risk of relapse and mortality of the treated cohorts as intermediate, high and very high-risk groups (corresponding to AJCC stages IIA, IIIA, or IIIB- IV), including deeper primary tumors in the intermediate risk category without nodal metastasis, and moving to those with resectable microscopic metastasis, and those with gross nodal 
metastasis or with resectable but limited extent of operable distant metastases in the highest risk group[3]. A durable impact on both relapse-free survival (RFS) and overall survival (OS) has been seen only with the regimen utilizing the high-dose IFN-a2b regimen (HDI) as tested in Eastern Cooperative Oncology Group (ECOG) and U.S. Intergroup trials E1684 $(\mathrm{N}=287), \mathrm{E} 1690(\mathrm{~N}=642), \mathrm{E} 1694(\mathrm{~N}=880)$ and E2696 $(\mathrm{N}=107)$ as adjuvant therapy for highrisk surgically resected melanoma (stage IIB, III or IV) [20-23]. The HDI regimen tested in these studies was administered in a 4-week induction phase given intravenously at 20 million IU $/ \mathrm{m}^{2} /$ day for five consecutive days out of seven every week $\times 4$ weeks, followed by a maintenance phase given subcutaneously at 10 million $\mathrm{IU} / \mathrm{m}^{2} /$ day every other day three times each week for an additional 48 weeks. All three phase III trials (E1684, E1690, E1694) demonstrated significant benefits in relation to RFS while significant benefits in relation to OS were seen in 2 of these trials. E1684, at a median follow up of 6.9 years, demonstrated significant relapse-free and overall survival prolongation with HDI versus observation (Obs) (median RFS 1.72 years versus 0.98 [ $(\mathrm{p}=0.0023])$, and median OS 3.82 versus 2.78 years [ $\mathrm{p}=0.0237])$ [20]. Patients with clinically node-negative but pathologically positive nodes (N1 disease) demonstrated the largest impact. The outcomes of this trial led to the regulatory approval by the United States Food and Drug Administration (FDA) in 1995. In E1694, the ganglioside GMK vaccine was selected as the optimal vaccine control arm at the time and this study reported significant improvements in both RFS (hazard ratio $[\mathrm{HR}]=1.47 ; \mathrm{p}=0.0015)$ and $\mathrm{OS}(\mathrm{HR}=1.52 ; \mathrm{p}=0.009)$ in favor of HDI; The intent-to-treat analysis (ITT) of this study showed similar benefits in RFS (HR=1.49) and OS (HR=1.38) [22]. In E1690, both the HDI regimen and a low-dose regimen of IFNa2b (LDI) at 3 MU SC thrice weekly for 2 years were compared to Obs. Accrual of patients in E1690 was completed between 1991 and 1995, and at 4.3 years median follow-up, the 5-year estimated RFS rates were $44 \%$ for HDI, $40 \%$ for LDI, and 35\% for the Obs arm, respectively.[21] The effect of HDI on RFS alone was able to reach statistical significance $(p=0.03)$. Neither HDI nor LDI was found to establish OS benefit compared with Obs (52\% high dose vs $53 \%$ low dose vs 55\% observation). However, improved OS of the E1690 Obs arm was noticed when compared to E1684 Obs arm (median 6 years vs 2.8 years). Unlike E1684, subjects in E1690 did not require elective lymph node dissection. More importantly, a retrospective analysis revealed some cross over from the observation arm at regional nodal recurrence to IFNa salvage therapy that may have impacted the survival analysis in E1690.

The ECOG led a randomized, phase II trial E2696 that recruited 107 patients with surgically resected stage IIB, III and IV disease that was conducted between 1998 and 2000.[23] The intent was to study the anti-GM2 antibody response to GMK vaccine in the presence versus absence of IFN. The study compared 3 arms - arm A (GMK plus concurrent HDI), arm B (GMK plus sequentially administered HDI), and arm C (GMK alone). The combined approach reduced the risk of recurrence when compared to GMK alone ( $\mathrm{HR}=1.96$ for $\mathrm{C}$ versus $B$ and $H R=1.75$ for $C$ versus $A$ ).

Pegylated IFNa (Peg-IFN) was tested in EORTC 18991 and achieved regulatory approval for use as adjuvant therapy of high-risk melanoma patients with lymph node metastases in the US in 2011 [24]. The covalent bonding of the interferon molecule with a polyethylene glycol moiety results in sustained absorption and longer half-life. The EORTC 18991 trial 
looked at the efficacy and safety of Peg-IFN versus observation amongst 1256 patients recruited from 2000 to 2002 with resected AJCC stage III melanoma. The regimen comprised induction doses of peg-IFN SC administered at $6 \mathrm{mcg} / \mathrm{kg}$ a week for 8 weeks, followed by maintenance doses of weekly SC injections at $3 \mathrm{mcg} / \mathrm{kg}$ for up to 5 years. At 7.6 years median follow-up, the group released data, which showed an improved RFS in the treatment arm (HR 0.87, 95\% CI $0.76-1.00, \mathrm{p}=0.05$ ) with no difference in OS/DMFS between observation and treatment group. Sub-set analysis indicated that subjects with microscopic nodal metastasis and ulcerated primary tumor had the greatest benefit in terms of RFS, OS and DMFS. During the study, peg-IFN was discontinued for toxicity in $37 \%$ of patients.

Therefore, significant reduction in the risk of melanoma recurrence was seen across multiple randomized controlled trials with IFN. A significant impact on the risk of death was only shown with HDI when compared to observation (E1684) and the GMK vaccine (E1694). PegIFN as tested in EORTC 18991 demonstrated RFS benefit that appears to be confined to patients with node positive disease.

\section{Inhibitors of immune checkpoints in the treatment of melanoma}

A number of immunotherapeutic strategies have been shown to increase the immune system's ability to control the progression and mortality of cancer but one of the most promising strategies to enhance the patient's antitumor response has been the use of monoclonal antibodies that block the immunoregulatory mechanisms that are able to suppress host responses to tumor associated antigens (TAAs). These immunomodulatory antibodies are now under intense recent investigation

\section{Blockade of cytotoxic T lymphocyte antigen-4 (CTLA-4)}

CTLA4 is a member of the CD28:B7 immunoglobulin superfamily and is normally expressed at low levels at the surface of naïve effector T-cells and regulatory T-cells (Tregs). After stimulation of naïve T cells through the T cell receptor (TCR), CTLA-4 localizes to the plasma membrane and competes with $\mathrm{CD} 28$ for B7, ultimately turning off $\mathrm{T}$ cell receptor signaling.[25] CTLA-4 is therefore a critical inhibitory check point, a molecule that down-regulates T-cell activation via a homeostatic feedback loop designed to prevent unwanted autoimmunity and establish tolerance to self-antigens.[26]

Anti-CTLA-4 monoclonal antibodies ipilimumab and tremelimumab are monoclonal antibodies that result in the blockade of CTLA-4 signaling prolonging T-cell activation, restoring T-cell proliferation, and thus amplifying T-cell-mediated immunity which enhances the patient's capacity to mount an antitumor immune response [26, 27].

Clinical testing of ipilimumab has now yielded significant new results and has been approved by United States Food and Drug Administration for the treatment of advanced melanoma. Two pivotal phase III trials with ipilimumab in advanced inoperable AJCC stage III and stage IV melanoma have been completed. One trial is a first line treatment comparison of combination therapy of ipilimumab administered at $10 \mathrm{mg} / \mathrm{kg}$ and dacarbazine versus dacarbazine and placebo. Results from this study showed that OS was 
significantly longer in previously untreated patients receiving ipilimumab plus DTIC than those receiving DTIC plus placebo (11.2 months versus 9.1 months; HR: 0.72, $\mathrm{P}<0.001$ ), with higher survival rates in the ipilimumab plus DTIC group at 1 year (47.3\% versus $36.3 \%), 2$ years (28.5\% versus $17.9 \%)$ and 3 years (20.8\% versus $12.2 \%$ ) [28]. The second trial tested the combination of ipilimumab at $3 \mathrm{mg} / \mathrm{kg}$ with or without gp100 peptide vaccine versus gp100 vaccine alone in the second line setting. This study randomized 676 pretreated patients and ipilimumab therapy was given at every 3 weeks for four doses without maintenance. However, responding patients were eligible for re-induction with ipilimumab if they relapsed. The 1-year and 2-year survival rates were 44\% (ipilimumab + gp 100), 46\% (ipilimumab + placebo), 25\% (ipilimumab + placebo) and 22\% (ipilimumab + gp100), 24\% (ipilimumab + placebo), $14 \%$ (gp100 + placebo). The best objective response rate was $5.7 \%$ (ipilimumab + gp100), 10.9\% (ipilimumab + placebo), 1.5\% (gp100 + placebo). Median OS increased from 6.4 months to 10.0 months with the addition of ipilimumab to gp100 vaccine (HR 0.68, $p<0.0001$ ) and long-term survival rates improved.[29] The derived survival benefits from ipilimumab in phase III trials for metastatic melanoma were unprecedented but at the same time, this therapeutic strategy presents new challenges in the management of unique immune mediated toxicities.

Similarly, tremelimumab showed promising clinical activity in earlier trial testing in advanced melanoma that led to a subsequent phase III clinical trial (A3671009) in patients with treatment-naive advanced melanoma. This open label study randomized patients to therapy with single-agent tremelimumab $(\mathrm{n}=328)$ or standard-of-care chemotherapy $(\mathrm{n}=$ 327 ) with either dacarbazine or temozolomide [30]. The primary endpoint was overall survival. At a second interim analysis, the Logrank test-statistic $(P=.729)$ crossed the prespecified O'Brien-Fleming futility boundary. Although the phase III study of tremelimumab for melanoma was closed for "futility", the 1-year survival rate of $>50 \%$ for tremelimumab and the median survival of 12.02 months (compared with 10.45 months for chemotherapy) were notable. In addition, the majority of responses to tremelimumab were durable. Factors related to the trial's eligibility criteria (limited by LDH) and its open label conduct, during the availability of alternate anti-CTLA4 strategies to which enrollees had access, and the salvage patterns of patients on the chemotherapy arm may have affected the survival analysis of this trial.

\section{PD-1 and PD-L1 as immunotherapeutic targets for melanoma}

Programmed cell death-1 (PD-1) is an immune-inhibitory receptor belonging to CD28/ CTLA4 receptor family expressed by activated $T$ and $B$ cells, [31-34] which binds to the two known ligands PD-L1 (B7-H1) [31-35] and PD-L2 (B7-DC).[36, 37] PD-1 negatively regulates $\mathrm{T}$ cell functions through the engagement of PD-L1, which is expressed by a wide variety of tissues [32-35]. PD-L1 is also expressed by human tumors, including melanoma, either constitutively or after treatment with IFN-a.[38,39] Numerous mechanisms of tumorinduced immunosuppression that contribute to the resistance of tumors to CTL responses have been well characterized,[40] and a number of experimental studies in animals and in vitro have clarified the role of PD-1/PDL1 interactions in inhibiting the effector functions of tumor-specific CD8+ T cells.[38, 39] 
High expression of PD-L1 on tumor cells has been found to correlate with poor prognosis and survival in various cancer types, including renal cell carcinoma, ovarian carcinoma (RCC) and melanoma [41-43]. However, most recent studies have shown that PD-L1 expression by metastatic melanoma correlates with the presence of tumor-infiltrating lymphocytes (TILs) in human melanocytic lesions where $98 \%$ of PD-L1+ tumors were associated with TILs compared with only $28 \%$ of PD-L1 (-) tumors. PD-L1 (+) melanocytes were almost always localized immediately adjacent to TILs.[44] Of further interest, interferon- $\gamma$, a primary inducer of PD-L1 expression, was detected at the interface of PD-L1 $(+)$ tumors and TILs, and absent in PD-L1 (-) tumors, suggesting that TILs trigger their own inhibition by secreting cytokines that drive tumor PD-L1 expression. Consistent with this hypothesis, overall survival of patients with PD-L1 (+) metastatic melanoma was significantly prolonged compared with that of patients with PD-L1 (-) metastatic melanoma. [44]

Multiple anti-PD-1 monoclonal antibodies have been developed for clinical applications and a number have now shown significant clinical activity in the management of advanced disease.

Nivolumab (also known as MDX-1106 and BMS-936558), is a fully human IgG4 anti-PD-1 blocking $\mathrm{mAb}$ that was tested in a phase I study in patients with refractory or relapsed solid tumors [34]. Therapy with anti-PD-1 has shown clinical efficacy in larger clinical trials involving patients with advancednon-small cell lung cancer, prostate cancer, renal cell cancer, colorectal cancer, and advanced melanoma [45]. Patients were treated every other week with anti-PD-1 antibody dose ranging from 0.1 to $10 \mathrm{mg} / \mathrm{kg}$ and response was assessed every 8 weeks. Nivolumab was tolerated up to $10 \mathrm{mg} / \mathrm{kg}$, the highest dose level tested, and no maximum tolerated dose (MTD) was identified.[46] Although the spectrum, frequency, and severity of nivolumab-related AEs was generally similar across the dose levels tested, the $10 \mathrm{mg} / \mathrm{kg}$ dose level had higher grade 3/4 drug-related SAEs and AEs leading to discontinuation. Grade 3 or 4 drug-related adverse events occurred in $14 \%$ of patients of whom three from pulmonary toxicity but no maximum tolerated dose (MTD) was defined. Adverse events consistent with immune-related causes were observed at an overall incidence of approximately $20 \%$. Most common were pruritus, skin rash, and diarrhea. Other immunerelated AEs (irAEs) include increase of TSH, increase of ALT/AST, pneumonitis, infusion reaction and vitiligo. Based upon the totality of the safety, efficacy, and exposure-response data, a dose of $3 \mathrm{mg} / \mathrm{kg}$ Q2W was selected as the dose anticipated to achieve an optimal level of efficacy with an appropriate risk for ongoing phase 2 clinical trials in advanced melanoma. Cumulative response rates of $28 \%$ (all doses) among patients with melanoma (26 of 94 patients) with response rate ranging from 19 to $41 \%$ by dose level. Notably, responses were durable and 13 of 18 responses lasted 1 year or more in melanoma patients with 1 year or more of follow-up. Immunohistochemical analysis of pretreatment tumor specimens show that patients with clinical response more often had PD-L1-positive tumors. Long-term survival of ipilimumab-naïve patients with advanced melanoma treated with nivolumab in a phase I trial was recently updated at the 2014 ASCO annual meeting. Median overall survival was 17.3 months across doses and 20.3 months at the $3 \mathrm{mg} / \mathrm{kg}$ dose. Survival rates were $63 \%$ at 1 year, $48 \%$ at 2 years, $41 \%$ at 3 years. Median PFS was 3.7 months across doses and 9.7 months at $3 \mathrm{mg} / \mathrm{kg}$. Further, 47 patients were still alive with a median overall 
follow-up of 22 months and a range of 14 to 51 months[47]. Phase II and III trials testing ipilimumab monotherapy and the combination with ipilimumab versus chemotherapy completed accrual in late 2013.

Pembrolizumab (previously known as SCH 900475, MK-3475 and Lambrolizumab) is a potent and highly selective humanized $\mathrm{mAb}$ of the IgG4/kappa isotype designed to block the interaction between PD-1 and its ligands, PD-L1 and PD-L2. An open label phase I study (Keynote Protocol 001) was designed to evaluate the safety and clinical activity of single agent pembrolizumab was completed in 411 patients. The dose escalation portion of this study evaluated three dose levels, $1 \mathrm{mg} / \mathrm{kg}, 3 \mathrm{mg} / \mathrm{kg}$, and $10 \mathrm{mg} / \mathrm{kg}$, administered every 2 weeks, in patients with advanced solid tumors. All three dose levels were well tolerated and no DLTs were observed. Based on PK data showing a half-life of 21 days, the protocol was amended to change the dosing frequency in the expansion cohort to every 3 weeks in ongoing clinical trials (Q3W). Preliminary evidence of antitumor activity was observed in melanoma and NSCLC. As of December 1, 2012, 294 patients with advanced melanoma have been enrolled, including 179 ipi-naïve and 115 ipi-pretreated. Patients were treated with pembrolizumab10 $\mathrm{mg} / \mathrm{kg}(\mathrm{n}=183)$ or $2 \mathrm{mg} / \mathrm{kg}(\mathrm{n}=111)$.[48] Preliminary data from the first 85 consecutive patients who were dosed before April 25, 2012 and had independent radiologic review available as of October 19, 2012 indicate a confirmed overall response rate (per RECIST 1.1) of more than 30\%. Responses have been durable, with only 2 patients who had an initial response discontinuing therapy due to disease progression. Of 133 patients who were dosed with pembrolizumab before July 31, 2012 and evaluable for AEs as of September 28, 2012, fatigue (22\%), rash (18\%), and pruritus (14\%) were the most common drug-related AEs (mostly grade 1/2). The incidence of drug-related grade $3 / 4$ AEs was $10 \%$ ( $24 \%$ regardless of attribution). There were 4 cases of drug-related pneumonitis, all grade $1 / 2$. Grade $3 / 4$ drug-related hypothyroidism $(n=1)$ and hyperthyroidism $(n=1)$ were noted. In a recent update presented at the 2013 ASCO Annual Meeting and published in NEJM, a total of 135 patients with advanced melanoma were treated.[48] Common adverse events attributed to treatment were fatigue, rash, pruritus, and diarrhea; most of the adverse events were low grade. The confirmed response rate across all dose cohorts, evaluated by central radiologic review according to the Response Evaluation Criteria in Solid Tumors (RECIST), version 1.1, was 38\% (95\% confidence interval [CI], 25 to 44), with the highest confirmed response rate observed in the cohort that received $10 \mathrm{mg}$ per kilogram every 2 weeks (52\%; 95\% CI, 38 to 66). The response rate did not differ significantly between patients who had received prior ipilimumab treatment and those who had not (confirmed response rate, 38\% [95\% CI, 23 to 55] and 37\% [95\% CI, 26 to 49], respectively). Responses were durable in the majority of patients (median follow-up, 11 months among patients who had a response); $81 \%$ of the patients who had a response (42 of 52) were still receiving treatment at the time of analysis in March 2013. The overall median progression-free survival among the 135 patients was longer than 7 months. [48] The results of this study were updated at the 2014 ASCO annual meeting. The overall response rate was $34 \%$, including $44 \%$ in treatment-naïve, $40 \%$ in ipilimumab-naïve and $28 \%$ in ipilimumabtreated patients. Responses were durable for all groups and $88 \%$ of responses were ongoing at the time of the report. Median duration of response was not reached (range, 6+ to 76+ 
weeks). Median PFS was 5.5 months and 69\% OS rate at 1 year (median OS not reached[49]).

Pidilizumab (previously CT-011) is a humanized IgG1 monoclonal antibody (mAb) that has been tested in phase I dose-escalation, single-dose $(0.2-6 \mathrm{mg} / \mathrm{kg})$ study of in patients with advanced hematologic malignancies ( $\mathrm{N}=17)$. [50] The maximum tolerated dose (MTD) was not achieved, and the doses tested were well-tolerated. This study has shown 1 complete response (NHL - follicular lymphoma), and 1 minimal response (AML) at the dose levels tested. [51] The results of a phase II study were presented at the 2014 ASCO annual meeting reporting a modest response rate of $6 \%$. The study missed the primary endpoint of objective response rate but met the secondary endpoint of overall survival where $64.5 \%$ of patients were alive at 12 months, although $57 \%$ of patients were treated with ipilimumab after progression on the study[52].

In addition to antibodies targeting PD-1, clinical antitumor activity has been observed with several anti-PD-L1 monoclonal antibodies. BMS-936559 (also known as MDX-1105) is a human IgG4 mAb that targets PD-L1, and was designed to disrupt the interaction of PD-L1 on tumor cells and PD-1 on effector T cells. BMS-936559 was tested in a dose escalation phase I/II study in pretreated patients with a variety of malignancies including NSCLC, melanoma, CRC, RCC, ovarian cancer, pancreatic cancer, and breast cancer[53]. The trial included 207 patients. Overall, potential irAEs were seen in $39 \%$ of patients. There were nine objective responses among 52 evaluable melanoma patients (17\%). Five of these had an objective response lasting at least one year, and six additional patients had stable disease that was maintained for at least 24 weeks. This antibody was the first published experience demonstrating the clinical efficacy of PD-L1 blockade.

MPDL3280A (Genentech, San Francisco, CA) is another monoclonal antibody that binds to PD1-L1 [54]. A phase I study in 45 patients with advanced melanoma reported an overall response rate of $29 \%$, and $43 \%$ of patients were progression-free at 24 weeks.

MEDI3740 (MedImmune, Gaithersburg, MD) is another antibody that targets PD-L1 now undergoing phase I clinical evaluation in a variety of malignancies. MSB0010718C (EMD Serono) is a further PD-L1 antibody that is undergoing dose escalation phase I trial with consecutive parallel group expansion in subjects with various tumors. This PD-L1 antibody is under clinical evaluation in metastatic colorectal cancer (mCRC), metastatic castrateresistant prostate cancer (mCRPC), melanoma and ovarian cancer. This study is currently recruiting participants, and results regarding its efficacy and safety are expected in the future.

\section{Other immune check points as immunotherapeutic targets}

Members of the tumor necrosis factor (TNF) superfamily are involved in the regulation of diverse immune functions. CD40 is a costimulatory molecule that is one such member that is widely expressed by immune cells and by cancer cells of various histologies. CD40 expression on immune cells has been implicated in the regulation of humoral and cellular immunity while CD40 expression by tumors has been implicated in pro-apoptotic and antiproliferative activity [55-58]. CD40 is broadly expressed on dendritic cells and its 
activation by CD40 ligand found on activated T cells appears to "license" the antigen presenting cell for T-cell activation [59]. CD40 stimulation leads to effective therapy of CD40 deficient tumors through strong induction of systemic cytotoxic T lymphocyte immunity [60] Therefore the targeting of CD40 may have antitumor effects indirectly through the activation of immune cells and/or directly by provoking tumor cell apoptosis and impaired tumor growth [55]. CP-870,893 (Pfizer) is a fully human IgG2 agonist mAb targeting CD40. Preclinical testing has shown its ability to activate dendritic cells and B cells, as well as antitumor activity in human xenograft models [61]. In a phase I dose escalation study of a single intravenous infusions in 29 patients, the single-dose MTD was estimated at $0.2 \mathrm{mg} / \mathrm{kg}$, with a dose-limiting cytokine-release syndrome associated with acute increase of serum levels of TNF- $\alpha$ and IL-6 and characterized clinically by fevers, chills, and rigors. This was associated with transient depletion in circulating CD40+CD19+ B cells and among B cells remaining in blood, a dose-related upregulation of costimulatory molecules (CD86) after treatment. This was also associated with the induction of melanoma antigen-specific $\mathrm{T}$ cells and clinically, objective partial responses were noted in four patients with metastatic melanoma [61]. A multiple dose phase I trial of weekly dosing of CP-870,893 for up to 8 doses was conducted in 27 patients. The MTD was again estimated at $0.2 \mathrm{mg} / \mathrm{kg}$ limited by a cytokine-release syndrome [62]. A phase I study testing the combination of CP-870,893 with carboplatin and paclitaxel in patients with solid tumors has been completed. A phase I study of CP-870,893 in combination with gemcitabine was conducted in patients with advanced pancreatic ductal adenocarcinoma. Twenty-two patients with chemotherapy-naïve advanced PDA were treated. The most common adverse event was cytokine release syndrome (grade 1 to 2). Four patients achieved a partial response (PR). Dacetuzumab (SGN-40) is a humanized IgG1 agonist mAb that also targets CD40 [63]. A phase I single dose study in patients $(\mathrm{N}=17)$ with lymphoid malignancies, acute myeloid leukemia and multiple myeloma demonstrated safety up to $6 \mathrm{mg} / \mathrm{kg}$ with no MTD declared. [64] A recent phase II study done in relapsed lymphoma (DLBCL) patients ( $N=46)$ evaluated the rate and duration of objective responses and safety of single-agent dacetuzumab. Forty-six adult patients with relapsed/refractory DLBCL received up to 12 cycles of intravenous dacetuzumab using intrapatient dose-escalation to a target dose of 8 $\mathrm{mg} / \mathrm{kg} /$ week in an initial 5 -week cycle, followed by 4-week cycles of $8 \mathrm{mg} / \mathrm{kg} /$ week. Overall response rate was $9 \%$ and disease control rate (complete remission + partial remission + stable disease) was $37 \%$. Common non-hematologic adverse events (AEs) included fatigue, headache, chills, fever, and nausea. The most frequent Grade 3-4 non-hematologic AE was deep venous thrombosis (3 patients). Dacetuzumab is in the most advanced stage and is being tested as treatment for malignancies such as chronic lymphatic leukemia (CLL), Multiple Myeloma (MM), and non-Hodgkin's lymphoma (NHL). The promising results from early clinical trials have encouraged clinical drug development in order to investigate the effect of CD40 mAbs in combination with other cancer immunotherapies, in particular interleukin (IL)-2.

OX40 and its ligand, OX40L, are key TNF family members that augment T-cell expansion, cytokine production, and survival. OX40 is best described as a costimulatory molecule that is expressed transiently at the surface of CD4+ and CD8+ T cells upon activation. OX40 is also expressed by $\mathrm{CD} 4+\mathrm{CD} 25+\mathrm{T}$ regulatory cells and controls regulatory T-cell 
differentiation and suppressive function. Engagement of OX40 on T regulatory cells appears to abrogate regulatory $\mathrm{T}$ cell suppressive function. [65-67] In murine models, engagement of OX40 in vivo with $\mathrm{Ab}$ agonist OX-40L:Ig during tumor priming has been shown to have antitumor activity. [68] A murine IgG1 agonist mAb targeting OX40 was tested in a phase I dose escalation trial. The trial was designed to test $0.1,0.4$, and $2 \mathrm{mg} / \mathrm{kg}$ dose levels administered on days 1,3 , and 5 in 3 cohorts of 10 patients each. The results of the first 2 cohorts testing 0.1 and $0.4 \mathrm{mg} / \mathrm{kg}$ of antibody have been reported at the 2009 ISBTC meeting with acceptable toxicity and 5 out of 20 patients having stable disease [69].

CD137 (4-1BB) and its ligand are members of the TNFR and TNF families, respectively, and are involved in the regulation of a wide range of immune activities. CD137 ligand crosslinks its receptor, $\mathrm{CD} 137$, which is expressed on activated $\mathrm{T}$ cells, and costimulates $\mathrm{T}$ cell activities. The co-stimulatory function induced by the cross-linking of CD137 on activated T cells enhances $\mathrm{T}$ cell proliferation and the memory and cytotoxic activity of T cells [70-72]. BMS-663513, a fully human anti-CD137 agonist monoclonal antibody that has been tested in a phase I dose escalation study [73]. Eighty thee patients (54 melanoma, 15 RCC, 13 ovarian and 1 prostate) were treated. Three responded and 4 had durable stable disease. A randomized, multi-dose phase II study of BMS-663513 as a second-line monotherapy in subjects with previously treated advanced melanoma has been completed. Two other studies tested combination with chemotherapy in solid tumors and chemoradiation in NSCLC [74].

\section{Combination therapy of interferon-alfa and immune checkpoint blockers}

\section{Anti-CTLA-4 and IFN}

The combined administration of anti CTLA-4 immunotherapy with tremelimumab and interferon alfa was recently examined in a phase II trial at our site, and has an acceptable safety profile and promising clinical activity in patients metastatic melanoma. The hypothesis driving this study was that that the combination of tremelimumab and interferon alfa- $2 \mathrm{~b}$ act via different and synergistic mechanisms that together would overcome tumor immune tolerance and lead to more significant and durable clinical benefits. In untreated advanced melanoma, the quality of the host immune response has been shown to be compromised, with strong bias toward melanoma antigen-specific Th2-type polarization [75], yielding a microenvironment that facilitates disease progression [76]. Strategies for overcoming tumor-induced immune suppression that build upon the success of IFN-a and its immunomodulatory qualities as demonstrated in the adjuvant setting [20] through downregulation of the CTLA4 suppressive regulatory elements are desirable [8]. IFN- $a$ at high dosage (HDI) has been shown to play a critical role in interrupting tumor immune tolerance both improving tumor immunogenicity and increasing dendritic cell (DC) activation and survival $[8,9]$. IFN-a upregulates major histocompatibility complex (MHC) antigen processing and costimulatory molecules leading to more efficient antigen presentation that may elicit previously low-affinity autoreactive T-cells [8-10]. Moreover, in their immature state, IFN-treated DCs induce a 'polarized' Th1 cytokine microenvironment [11]. Similarly, type-I IFNs polarize lymphocytes towards the pro-inflammatory Th1 phenotype [12-14]. This significant impact of type-I IFNs in the cytotoxic T-cell compartment, induces potent antitumor cell-mediated cytotoxicity,[15] and promotes natural 
killer (NK) cell-mediated proliferation and cytotoxicity[77] The IFN-induced Th1 bias can be detected in the circulating blood of melanoma patients as upregulated pro-inflammatory cytokine response (Th1 polarization) as we have previously demonstrated in the context of the adjuvant E1694 trial [16]. In addition, locally produced type-I IFNs induce the expression of integrins and chemokine receptors and recruitment of natural killer cells and macrophages leading to Th1 rather than Th2 lymphocyte traffic to the tumor site [17]. This has been demonstrated clinically where responding patients had significantly greater increases in intra-tumor CD11c+ DCs and CD3+ T-cells in a neoadjuvant melanoma study of HDI [18].

This potent anti-tumor impact of IFN-a can be suppressed by tolerogenic mechanisms of the tumor, and may in part explain the limited clinical activity of IFN-a as a monotherapy in metastatic melanoma. Combination with CTLA-4 blockade may alter this balance, downregulating CTLA4-mediated counter regulatory mechanisms and possibly also releasing inhibitory influences on activated CD4 and CD8 effector cells. CTLA4 is a key element in immune tolerance and a central negative regulator of $\mathrm{T}$ cell-mediated antitumor immune responses where preclinical studies suggested that it serves as a natural braking mechanism for T-cell activation [78-81]. The inhibitory signal produced by CTLA4 is therefore blocked by anti-CTLA4 antibodies (tremelimumab or ipilimumab), and T-cell activation is enhanced [82-86]. Tremelimumab has been demonstrated to have a significant immune modulating role, unlocking the immune response by disrupting CTLA-4, enhancing pro-inflammatory T-cell cytokine production[87], and increasing T-cell infiltration in responding tumors [88]. Therefore, it was hypothesized that IFN- $a$ and tremelimumab may have additive or synergistic effects in promoting tumor elimination.

In the phase II study that tested the safety and efficacy of the combination of HDI and tremelimumab, tremelimumab $15 \mathrm{mg} / \mathrm{kg}$ was administered intravenously every 12 weeks and IFN-a-2b was administered intravenously at $20 \mathrm{MU} / \mathrm{m}^{2} / \mathrm{d}$ for $5 \mathrm{~d} / \mathrm{wk}$ for 4 weeks, then subcutaneously at $10 \mathrm{MU} / \mathrm{m}^{2} / \mathrm{d}$ for $3 \mathrm{~d} / \mathrm{wk}$ for 8 weeks. Patients without evidence of PD or limiting toxicities were offered additional courses of therapy up to a maximum of four courses where IFN- $a-2 b$ was administered only subcutaneously. Thirty-seven patients with stage IV (9M1a, 6M1b, and 22M1c) melanoma were enrolled. Two patients had previously treated brain metastases. Grades 3 and 4 toxicities included neutropenia (six patients; 17\%), diarrhea/colitis (four patients; 11\%), liver enzyme increase (four patients; 11\%), rash (four patients; $11 \%$ ), fatigue (15 patients; 40\%), and anxiety/depression (five patients; $14 \%$ ). Response data were available for 35 patients. The best objective response rate analyzed by intention to treat was $24 \%$ (90\% CI, $13 \%$ to $36 \%$, with four complete responses [CRs] and five partial responses [PRs] that lasted $6,6,>12,>14,>18,20,>28,30$, and $>37$ months, respectively. Fourteen patients (38\%) had stable disease (SD) that lasted 1.5 to 21 months. The median progression-free survival was 6.4 months (95\% CI, 3.3 to 12.1 months). The median overall survival (OS) was 21 months (95\% CI, 9.5 to not reached). There was a weak association between therapy-induced autoimmunity and clinical benefits (CR/PR/SD; $P=.0059)$, baseline C-reactive protein (CRP) less than or equal to $2.7 \times$ the upper limit of normal and clinical benefits $(P=.0494)$ and improved probability of survival $(P=.0032)$, and baseline lymphocyte count of at least $1,000 / \mu \mathrm{L}$ and response $(\mathrm{CR} / \mathrm{PR} ; P=.0183)$ and 
clinical benefits $(\mathrm{CR} / \mathrm{PR} / \mathrm{SD} ; P=.0255)$. Biomarker associations were not significant after adjustment for multiple comparisons [89]. Additional exploratory analysis was conducted utilizing the model proposed by Korn et al, to calculate an expected one year survival rate based on the patients' baseline characteristics including performance status, gender, visceral disease and the fact that the study enrolled subjects with treated brain metastases. The predicted 1-year OS rate was $21 \%$ which was significantly worse than the observed rate of $62 \%$ (95\% CI, $46 \%-78 \%$ ); $\mathrm{p}<0.0001$. Immune monitoring studies within this trial showed significant downregulation of myeloid derived suppressor cells after initiation of the therapy suggesting a role in the significant clinical activity observed with this regimen [90].

Currently, the potential immunomodulatory and antitumor effects of the combination of ipilimumab and IFN-a2b are being tested in 2 ongoing trials. UPCI 11-063 is a biomarker driven mechanistic study designed to evaluate immunomodulatory effects of this combination in the tumor tissues and blood of patients with stage IIIB-C melanoma, and secondarily to evaluate the safety and potential efficacy of the combination as neoadjuvant therapy before definitive surgery. The study provides access to tumor and blood before and at the close of 6 weeks neoadjuvant treatment, allowing for novel mechanistic and biomarker studies. This study is expected to complete accrual in the second half of 2014, and will also provide important early information on the antitumor efficacy of this combination. E3611 is a randomized phase II trial of the ECOG-ACRIN national cooperative group, testing the antitumor efficacy of the combination of ipilimumab and HDI in advanced inoperable metastatic melanoma. The study has a $2 \times 2$ factorial design that allows the evaluation of HDI in combination with ipilimumab at the dose levels of $3 \mathrm{mg} / \mathrm{kg}$ and $3 \mathrm{mg} / \mathrm{kg}$ versus monotherapy with ipilimumab.

\section{Anti-PD1 and IFN}

Because IFNa and other cytokines induce the expression of PDL1, and because expression of PDL1 has shown potential predictive role in anti-PD1 antibody therapy as previously reviewed, there has been interest in the antitumor benefits of the combination of IFN and anti-PD1 therapy. The combination of pembrolizumab and peginterferon alfa- $2 b$ may increase tumor-infiltrating CD8+ T cells, PD-1 expression by TILs, as well as PD-L1 expression by melanoma cells-culminating in greater inhibition of tumor growth and increased tumor cell apoptosis, as well as increased expression of HLA-class I antigen presenting machinery components and HLA class I antigen-melanoma derived peptide complex expression by melanoma cells. IFNa is known to upregulate the expression of major histocompatibility complex (MHC) antigens, as well as antigen-processing and costimulatory molecules, leading to more efficient antigen presentation that may elicit previously low-affinity autoreactive $\mathrm{T}$-cells, $[8,9]$ that guide cytotoxic $\mathrm{T}$ lymphocytesmediated cellular killing.[91] Similarly, IFNa polarizes lymphocytes towards the proinflammatory Th1 phenotype,[12-14] promoting a significant impact of type I IFNs in the cytotoxic $\mathrm{T}$ cell compartment, inducing potent antitumor cell-mediated cytotoxicity[15], and promoting natural killer (NK) cell-mediated proliferation and cytotoxicity.[77] In addition, Type I IFNs have been reported to provide a "third signal" to CD8+ T cells, activating them directly, stimulating their clonal expansion and differentiation.[92] On the other hand, Gerner et al., have reported that CD8+ T cells matured in the presence of IFNa appear to re- 
express significantly higher levels of PD-1 upon antigen restimulation than IL-12 matured T cells and have shown a relatively poor ability of IFNa-matured $\mathrm{T}$ cells to efficiently inhibit tumor growth that appears to be largely due to PD-1 upregulation.[93] In addition, Fourcade et al., reported that tumor antigen-specific CD8+ T cells up-regulate PD-1 expression both in the circulation and in the tumor microenvironment and PD-1 blockade can enhance the expansion of tumor antigen-specific CD8+ T cells.[94] Further, IFNa has been reported to markedly augment PD-L1 cellular expression.[95] Iwai et al., have reported that transgenic expression of PD-L1 in P815 tumor cells rendered them less susceptible to the specific T cell antigen receptor-mediated lysis by cytotoxic $\mathrm{T}$ cells in vitro, and markedly enhanced their tumorigenesis and invasiveness in vivo in the syngeneic hosts as compared with the parental tumor cells that lacked endogenous PD-L. Both effects could be reversed by antiPD-L1 Ab.[39] These data support our hypothesis that IFNa-mediated antitumor activity would be significantly enhanced through PD-1 blockade. A phase I trial has recently been proposed and initiated at the University of Pittsburgh Cancer Institute that will evaluate the combination of pegylated IFN alfa-2b with pembrolizumab, and this trial UPCI 13-105 will test pembrolizumab at the proposed optimal dosage of $2 \mathrm{mg} / \mathrm{kg}$ every 3 weeks with and pegIFN at weekly dosages of 1,2 , or $3 \mathrm{mcg} / \mathrm{kg}$ in patients with metastatic melanoma. Building on our efforts in the neoadjuvant seeting, UPCI 14-102 will test the combination of pembrolizumab and high dose IFNa (HDI) in patients with regionally advanced operable and biopsiable disease. This neoadjuvant trial that is designed as a biomarker-driven and mechanistic study is expected to be activated in the last quarter of 2014.

\section{Conclusion}

IFNa has significant immunomodulatory and anti-tumor clinical activity that play a role in its antitumor efficacy in metastatic melanoma, and its adjuvant efficacy in $>20$ phase III trials as reviewed here. However, the antitumor efficacy of this immunotherapy agent in metastatic disease appears to be limited by tumor-induced immunosuppressive mechanisms that are greater over the spectrum of tumor progression from primary to regional and distant metastatic disease. Overcoming tumor-induced immune suppression has been increasingly possible through strategies that may build upon the immunomodulatory and clinical benefits of interferon- $a$, first in metastatic disease and then in the adjuvant setting where the burden of disease and the clinical needs to improve the efficacy of our therapy is even larger. Immunologic checkpoints are critical components of the immune system, that function in an elaborate system of self-regulation that in normal circumstances apart from neoplasia may prevent immune responses to our skin and GI epithelial barriers, and the protein synthetic organ of the liver and the endocrine systems. Manipulation of the effects of the checkpoints that serve in the absence of cancer to damp the capacity to respond to novel antigens may, in the setting of malignant neoplasms like melanoma allow therapeutic antibodies to control or even eliminate cancers.. Antibodies that block inhibitory pathways, such as those directed against CTLA-4, PD-1 and PD-L1 developed for clinical use against cancer are logical companions to the cytokine/IFN response system, where additive or synergistic effects may be realized. These checkpoint-blocking antibodies are revitalizing interest in solid tumor immunotherapy and have resulted in promising clinical outcomes in various malignancies as a monotherapy and in combination with other immunotherapeutic agents. The combined 
administration of anti CTLA-4 immunotherapy with tremelimumab plus high-dose IFN has resulted in durable antitumor efficacy that is promising in relation to current trials of the national cooperative groups testing the combination of anti-CTLA4 blocking antibody ipilimumab with IFN, and new trials testing the anti-PD1 antibody pembrolizumab and IFN in both metastatic disease, and the neoadjuvant setting. While evaluations of immunotherapy have largely focused on melanoma to date, there is growing evidence that this approach has benefits in other solid tumors such as bronchogenic non-small cell cancers.

\section{REFERENCES}

1. Siegel R, Ma J, Zou Z, Jemal A. Cancer statistics, 2014. CA Cancer J Clin. 2014; 64:9-29. [PubMed: 24399786]

2. SEER Cancer Statistics Review, 1975. 2009 (Vintage 2009 Populations. [http://seercancergov/csr/ 1975_2009_pops09/ (based on November 2011 SEER datasubmission, posted to the SEER wevsite April 2012.]

3. Tarhini AA, Kirkwood JM. Clinical and immunologic basis of interferon therapy in melanoma. Ann N Y Acad Sci. 2009; 1182:47-57. [PubMed: 20074274]

4. Kotenko SV, Gallagher G, Baurin VV, Lewis-Antes A, Shen M, Shah NK, et al. IFN-lambdas mediate antiviral protection through a distinct class II cytokine receptor complex. Nat Immunol. 2003; 4:69-77. [PubMed: 12483210]

5. Sheppard P, Kindsvogel W, Xu W, Henderson K, Schlutsmeyer S, Whitmore TE, et al. IL-28, IL-29 and their class II cytokine receptor IL-28R. Nat Immunol. 2003; 4:63-68. [PubMed: 12469119]

6. O'Brien TR. Interferon-alfa, interferon-lambda and hepatitis C. Nat Genet. 2009; 41:1048-1050. [PubMed: 19749756]

7. Platanias LC. Mechanisms of type-I- and type-II-interferon-mediated signalling. Nat Rev Immunol. 2005; 5:375-386. [PubMed: 15864272]

8. Kirkwood JM, Richards T, Zarour HM, Sosman J, Ernstoff M, Whiteside TL, et al. Immunomodulatory effects of high-dose and low-dose interferon alpha2b in patients with high-risk resected melanoma: the E2690 laboratory corollary of intergroup adjuvant trial E1690. Cancer. 2002; 95:1101-1112. [PubMed: 12209697]

9. Wang W, Edington HD, Rao UN, Jukic DM, Land SR, Ferrone S, et al. Modulation of signal transducers and activators of transcription 1 and 3 signaling in melanoma by high-dose IFNalpha2b. Clin Cancer Res. 2007; 13:1523-1531. [PubMed: 17332298]

10. Paquette RL. Interferon-alpha and granulocyte-macrophage colony-stimulating factor differentiate peripheral blood monocytes into potent antigen-presenting cells. J Leukoc Biol. 1998; 64:358-367. [PubMed: 9738663]

11. Parlato S, Santini SM, Lapenta C, Di Pucchio T, Logozzi M, Spada M, et al. Expression of CCR-7, MIP-3beta, and Th-1 chemokines in type I IFN-induced monocyte-derived dendritic cells: importance for the rapid acquisition of potent migratory and functional activities. Blood. 2001; 98:3022-3029. [PubMed: 11698286]

12. Brinkmann V, Geiger T, Alkan S, Heusser CH. Interferon alpha increases the frequency of interferon gamma-producing human CD4+ T cells. J Exp Med. 1993; 178:1655-1663. [PubMed: 8228812]

13. Wenner CA, Guler ML, Macatonia SE, O'Garra A, Murphy KM. Roles of IFN-gamma and IFNalpha in IL-12-induced T helper cell-1 development. J Immunol. 1996; 156:1442-1447. [PubMed: 8568246]

14. Rogge L, Barberis-Maino L, Biffi M, Passini N, Presky DH, Gubler U, et al. Selective expression of an interleukin-12 receptor component by human T helper 1 cells. J Exp Med. 1997; 185:825831. [PubMed: 9120388]

15. Palmer KJ, Harries M, Gore ME, Collins MK. Interferon-alpha (IFN-alpha) stimulates antimelanoma cytotoxic T lymphocyte (CTL) generation in mixed lymphocyte tumour cultures (MLTC). Clin Exp Immunol. 2000; 119:412-418. [PubMed: 10691911] 
16. Yurkovetsky ZR, Kirkwood JM, Edington HD, Marrangoni AM, Velikokhatnaya L, Winans MT, et al. Multiplex analysis of serum cytokines in melanoma patients treated with interferon-alpha2b. Clin Cancer Res. 2007; 13:2422-2428. [PubMed: 17438101]

17. Tough DF. Type I interferon as a link between innate and adaptive immunity through dendritic cell stimulation. Leuk Lymphoma. 2004; 45:257-264. [PubMed: 15101709]

18. Moschos SJ, Edington HD, Land SR, Rao UN, Jukic D, Shipe-Spotloe J, et al. Neoadjuvant treatment of regional stage IIIB melanoma with high-dose interferon alfa-2b induces objective tumor regression in association with modulation of tumor infiltrating host cellular immune responses. J Clin Oncol. 2006; 24:3164-3171. [PubMed: 16809739]

19. Fuertes MB, Kacha AK, Kline J, Woo SR, Kranz DM, Murphy KM, et al. Host type I IFN signals are required for antitumor CD8+ T cell responses through CD8 $\{$ alpha $\}+$ dendritic cells. J Exp Med. 2011; 208:2005-2016. [PubMed: 21930765]

20. Kirkwood JM, Strawderman MH, Ernstoff MS, Smith TJ, Borden EC, Blum RH. Interferon alfa-2b adjuvant therapy of high-risk resected cutaneous melanoma: the Eastern Cooperative Oncology Group Trial EST 1684. J Clin Oncol. 1996; 14:7-17. [PubMed: 8558223]

21. Kirkwood JM, Ibrahim JG, Sondak VK, Richards J, Flaherty LE, Ernstoff MS, et al. High- and low-dose interferon alfa-2b in high-risk melanoma: first analysis of intergroup trial E1690/S9111/ C9190. J Clin Oncol. 2000; 18:2444-2458. [PubMed: 10856105]

22. Kirkwood JM, Ibrahim JG, Sosman JA, Sondak VK, Agarwala SS, Ernstoff MS, et al. High-dose interferon alfa-2b significantly prolongs relapse-free and overall survival compared with the GM2KLH/QS-21 vaccine in patients with resected stage IIB-III melanoma: results of intergroup trial E1694/S9512/C509801. J Clin Oncol. 2001; 19:2370-2380. [PubMed: 11331315]

23. Kirkwood JM, Ibrahim J, Lawson DH, Atkins MB, Agarwala SS, Collins K, et al. High-dose interferon alfa- $2 b$ does not diminish antibody response to GM2 vaccination in patients with resected melanoma: results of the Multicenter Eastern Cooperative Oncology Group Phase II Trial E2696. J Clin Oncol. 2001; 19:1430-1436. [PubMed: 11230488]

24. Eggermont AM, Suciu S, Testori A, Santinami M, Kruit WH, Marsden J, et al. Long-term results of the randomized phase III trial EORTC 18991 of adjuvant therapy with pegylated interferon alfa-2b versus observation in resected stage III melanoma. J Clin Oncol. 2012; 30:3810-3818. [PubMed: 23008300]

25. Linsley PS, Bradshaw J, Greene J, Peach R, Bennett KL, Mittler RS. Intracellular trafficking of CTLA-4 and focal localization towards sites of TCR engagement. Immunity. 1996; 4:535-543. [PubMed: 8673700]

26. Peggs KS, Quezada SA, Korman AJ, Allison JP. Principles and use of anti-CTLA4 antibody in human cancer immunotherapy. Curr Opin Immunol. 2006; 18:206-213. [PubMed: 16464564]

27. Robert C, Ghiringhelli F. What is the role of cytotoxic T lymphocyte-associated antigen 4 blockade in patients with metastatic melanoma? Oncologist. 2009; 14:848-861. [PubMed: 19648604]

28. Robert C, Thomas L, Bondarenko I, O'Day S, M DJ, Garbe C, et al. Ipilimumab plus dacarbazine for previously untreated metastatic melanoma. N Engl J Med. 2011; 364:2517-2526. [PubMed: 21639810]

29. Hodi FS, O'Day SJ, McDermott DF, Weber RW, Sosman JA, Haanen JB, et al. Improved survival with ipilimumab in patients with metastatic melanoma. N Engl J Med. 2010; 363:711-723. [PubMed: 20525992]

30. Ribas A, Hauschild A, Kefford R, Punt CJ, Haanen JB, Marmol M, et al. Phase III, open-label, randomized, comparative study of tremelimumab (CP-675,206) and chemotherapy (temozolomide [TMZ] or dacarbazine [DTIC]) in patients with advanced melanoma (May 20 Supplement). J Clin Oncol. 2008; 26 Abstract LBA9011.

31. Chen L. Co-inhibitory molecules of the B7-CD28 family in the control of T-cell immunity. Nat Rev Immunol. 2004; 4:336-347. [PubMed: 15122199]

32. Freeman GJ, Long AJ, Iwai Y, Bourque K, Chernova T, Nishimura H, et al. Engagement of the PD-1 immunoinhibitory receptor by a novel B7 family member leads to negative regulation of lymphocyte activation. J Exp Med. 2000; 192:1027-1034. [PubMed: 11015443]

33. Okazaki T, Iwai Y, Honjo T. New regulatory co-receptors: inducible co-stimulator and PD-1. Curr Opin Immunol. 2002; 14:779-782. [PubMed: 12413529] 
34. Sharpe AH, Wherry EJ, Ahmed R, Freeman GJ. The function of programmed cell death 1 and its ligands in regulating autoimmunity and infection. Nat Immunol. 2007; 8:239-245. [PubMed: 17304234]

35. Dong H, Zhu G, Tamada K, Chen L. B7-H1, a third member of the B7 family, co-stimulates T-cell proliferation and interleukin-10 secretion. Nat Med. 1999; 5:1365-1369. [PubMed: 10581077]

36. Tseng SY, Otsuji M, Gorski K, Huang X, Slansky JE, Pai SI, et al. B7-DC, a new dendritic cell molecule with potent costimulatory properties for T cells. J Exp Med. 2001; 193:839-846. [PubMed: 11283156]

37. Latchman Y, Wood CR, Chernova T, Chaudhary D, Borde M, Chernova 1, et al. PD-L2 is a second ligand for PD-1 and inhibits T cell activation. Nat Immunol. 2001; 2:261-268. [PubMed: 11224527]

38. Blank C, Brown I, Peterson AC, Spiotto M, Iwai Y, Honjo T, et al. PD-L1/B7H-1 inhibits the effector phase of tumor rejection by T cell receptor (TCR) transgenic CD8+ T cells. Cancer Res. 2004; 64:1140-1145. [PubMed: 14871849]

39. Iwai Y, Ishida M, Tanaka Y, Okazaki T, Honjo T, Minato N. Involvement of PD-L1 on tumor cells in the escape from host immune system and tumor immunotherapy by PD-L1 blockade. Proc Natl Acad Sci U S A. 2002; 99:12293-12297. [PubMed: 12218188]

40. Gajewski TF. Identifying and overcoming immune resistance mechanisms in the melanoma tumor microenvironment. Clin Cancer Res. 2006; 12:2326s-2330s. [PubMed: 16609053]

41. Hamanishi J, Mandai M, Iwasaki M, Okazaki T, Tanaka Y, Yamaguchi K, et al. Programmed cell death 1 ligand 1 and tumor-infiltrating CD8+ T lymphocytes are prognostic factors of human ovarian cancer. Proc Natl Acad Sci U S A. 2007; 104:3360-3365. [PubMed: 17360651]

42. Thompson RH, Kuntz SM, Leibovich BC, Dong H, Lohse CM, Webster WS, et al. Tumor B7-H1 is associated with poor prognosis in renal cell carcinoma patients with long-term follow-up. Cancer Res. 2006; 66:3381-3385. [PubMed: 16585157]

43. Hino R, Kabashima K, Kato Y, Yagi H, Nakamura M, Honjo T, et al. Tumor cell expression of programmed cell death-1 ligand 1 is a prognostic factor for malignant melanoma. Cancer. 2010; 116:1757-1766. [PubMed: 20143437]

44. Taube JM, Anders RA, Young GD, Xu H, Sharma R, McMiller TL, et al. Colocalization of inflammatory response with B7-h1 expression in human melanocytic lesions supports an adaptive resistance mechanism of immune escape. Sci Transl Med. 2012; 4:127ra137.

45. Topalian SL, Hodi FS, Brahmer JR, Gettinger SN, Smith DC, McDermott DF, et al. Safety, activity, and immune correlates of anti-PD-1 antibody in cancer. N Engl J Med. 2012; 366:24432454. [PubMed: 22658127]

46. Brahmer JR, Drake CG, Wollner I, Powderly JD, Picus J, Sharfman WH, et al. Phase I study of single-agent anti-programmed death-1 (MDX-1106) in refractory solid tumors: safety, clinical activity, pharmacodynamics, and immunologic correlates. J Clin Oncol. 2010; 28:3167-3175. [PubMed: 20516446]

47. Hodi, F.; Sznol, M.; Kluger, H.; McDermott, D.; Carvajal, R.; Lawrence, D., et al. 2014 ASCO Annual Meeting; J Clin Oncol. Chicago, IL: 2014. Long-term survival of ipilimumab-naive patients (pts) with advanced melanoma (MEL) treated with nivolumab (anti-PD-1, BMS-936558, ONO-4538) in a phase I trial. abstract 9002;

48. Hamid O, Robert C, Daud A, Hodi FS, Hwu WJ, Kefford R, et al. Safety and tumor responses with lambrolizumab (anti-PD-1) in melanoma. N Engl J Med. 2013; 369:134-144. [PubMed: 23724846]

49. Ribas, A.; Hodi, F.; Kefford, R.; Hamis, O.; Daud, A.; Wolchok, J., et al. 2014 ASCO Annual Meeting; J Clin Oncol. Chicago, IL: 2014. Efficacy and safety of the anti-PD-1 monoclonal antibody MK-3475 in 411 patients (pts) with melanoma (MEL). abstract LBA9000

50. Berger R, Rotem-Yehudar R, Slama G, Landes S, Kneller A, Leiba M, et al. Phase I safety and pharmacokinetic study of CT-011, a humanized antibody interacting with PD-1, in patients with advanced hematologic malignancies. Clin Cancer Res. 2008; 14:3044-3051. [PubMed: 18483370]

51. ClinicalTrials.gov. [http://www.clinicaltrials.gov/ct2/results?term=CT-011+.] 
52. Atkins, M.; Kudchadkar, R.; Sznol, M.; McDermott, D.; Lotem, M.; Schachter, J., et al. 2014 ASCO Annual Meeting; J Clin Oncol. Chicago, IL: 2014. Phase 2, multicenter, safety and efficacy study of pidlilizumab in patients with metastatic melanoma. abstract 9001 ;

53. Brahmer JR, Tykodi SS, Chow LQ, Hwu WJ, Topalian SL, Hwu P, et al. Safety and activity of anti-PD-L1 antibody in patients with advanced cancer. N Engl J Med. 2012; 366:2455-2465. [PubMed: 22658128]

54. Herbst RS, Gordon MS, Fine GD, Sosman JA, Soria JC, Hamid O, et al. A study of MPDL3280A, an engineered PD-L1 antibody in patients with locally advanced or metastatic tumors. In J Clin Oncol. 2013

55. Fonsatti E, Maio M, Altomonte M, Hersey P. Biology and clinical applications of CD40 in cancer treatment. Semin Oncol. 2010; 37:517-523. [PubMed: 21074067]

56. Sotomayor EM, Borrello I, Tubb E, Rattis FM, Bien H, Lu Z, et al. Conversion of tumor-specific CD4+ T-cell tolerance to T-cell priming through in vivo ligation of CD40. Nat Med. 1999; 5:780 787. [PubMed: 10395323]

57. Diehl L, den Boer AT, Schoenberger SP, van der Voort EI, Schumacher TN, Melief CJ, et al. CD40 activation in vivo overcomes peptide-induced peripheral cytotoxic T-lymphocyte tolerance and augments anti-tumor vaccine efficacy. Nat Med. 1999; 5:774-779. [PubMed: 10395322]

58. Pan PY, Ma G, Weber KJ, Ozao-Choy J, Wang G, Yin B, et al. Immune stimulatory receptor $\mathrm{CD} 40$ is required for $\mathrm{T}$-cell suppression and $\mathrm{T}$ regulatory cell activation mediated by myeloidderived suppressor cells in cancer. Cancer Res. 2010; 70:99-108. [PubMed: 19996287]

59. van Mierlo GJ, Boonman ZF, Dumortier HM, den Boer AT, Fransen MF, Nouta J, et al. Activation of dendritic cells that cross-present tumor-derived antigen licenses CD8+ CTL to cause tumor eradication. J Immunol. 2004; 173:6753-6759. [PubMed: 15557168]

60. van Mierlo GJ, den Boer AT, Medema JP, van der Voort EI, Fransen MF, Offringa R, et al. CD40 stimulation leads to effective therapy of CD40(-) tumors through induction of strong systemic cytotoxic T lymphocyte immunity. Proc Natl Acad Sci U S A. 2002; 99:5561-5566. [PubMed: 11929985]

61. Vonderheide RH, Flaherty KT, Khalil M, Stumacher MS, Bajor DL, Hutnick NA, et al. Clinical activity and immune modulation in cancer patients treated with CP-870,893, a novel CD40 agonist monoclonal antibody. J Clin Oncol. 2007; 25:876-883. [PubMed: 17327609]

62. Ruter J, Antonia SJ, Burris HA, Huhn RD, Vonderheide RH. Immune modulation with weekly dosing of an agonist CD40 antibody in a phase I study of patients with advanced solid tumors. Cancer Biol Ther. 2010; 10:983-993. [PubMed: 20855968]

63. Law CL, Gordon KA, Collier J, Klussman K, McEarchern JA, et al. Preclinical antilymphoma activity of a humanized anti-CD40 monoclonal antibody, SGN-40. Cancer Res. 2005; 65:83318338. [PubMed: 16166310]

64. Khubchandani S, Czuczman MS, Hernandez-Ilizaliturri FJ. Dacetuzumab, a humanized mAb against CD40 for the treatment of hematological malignancies. Curr Opin Investig Drugs. 2009; 10:579-587.

65. Redmond WL, Gough MJ, Charbonneau B, Ratliff TL, Weinberg AD. Defects in the acquisition of CD8 T cell effector function after priming with tumor or soluble antigen can be overcome by the addition of an OX40 agonist. J Immunol. 2007; 179:7244-7253. [PubMed: 18025166]

66. Kjaergaard J, Tanaka J, Kim JA, Rothchild K, Weinberg A, Shu S. Therapeutic efficacy of OX-40 receptor antibody depends on tumor immunogenicity and anatomic site of tumor growth. Cancer Res. 2000; 60:5514-5521. [PubMed: 11034096]

67. Evans DE, Prell RA, Thalhofer CJ, Hurwitz AA, Weinberg AD. Engagement of OX40 enhances antigen-specific CD4(+) T cell mobilization/memory development and humoral immunity: comparison of alphaOX-40 with alphaCTLA-4. J Immunol. 2001; 167:6804-6811. [PubMed: 11739496]

68. Weinberg AD, Rivera MM, Prell R, Morris A, Ramstad T, Vetto JT, et al. Engagement of the OX-40 receptor in vivo enhances antitumor immunity. J Immunol. 2000; 164:2160-2169. [PubMed: 10657670] 
69. Kovacsovics-Bankowski M, Walker E, K F. Increased CD4 and CD8 memory T cell proliferation following anti-OX40 administration to cancer patients: immunologic assessment of a phase I clinical trial. In ISBTC. 2009

70. Zhang H, Snyder KM, Suhoski MM, Maus MV, Kapoor V, June CH, et al. 4-1BB is superior to $\mathrm{CD} 28$ costimulation for generating CD8+ cytotoxic lymphocytes for adoptive immunotherapy. $\mathrm{J}$ Immunol. 2007; 179:4910-4918. [PubMed: 17878391]

71. Miller RE, Jones J, Le T, Whitmore J, Boiani N, Gliniak B, et al. 4-1BB-specific monoclonal antibody promotes the generation of tumor-specific immune responses by direct activation of CD8 T cells in a CD40-dependent manner. J Immunol. 2002; 169:1792-1800. [PubMed: 12165501]

72. May KF Jr, Chen L, Zheng P, Liu Y. Anti-4-1BB monoclonal antibody enhances rejection of large tumor burden by promoting survival but not clonal expansion of tumor-specific CD8+ T cells. Cancer Res. 2002; 62:3459-3465. [PubMed: 12067989]

73. Sznol M, Hodi FS, Margolin K. Phase I study of BMS-663513, a fully human anti-CD137 agonist monoclonal antibody, in patients (pts) with advanced cancer (CA). 2008

74. ClinicalTrials.gov. [http://www.clinicaltrials.gov/ct2/results? term $=$ BMS- $666513 \&$ recr $=\& r s i t=\&$ ttoe $=\& \mathrm{cond}=\& i n t r=\&$ outc $=\& l e a d=\&$ spons $=\& i d=\&$ state $1=\& \mathrm{cn}$ try $1=\&$ state $2=\&$ cntry $2=\&$ state $3=\&$ cntry $3=\& l o c n=\& q n d r=\&$ rcv s=\&rcv e=\&lup s=lup e=.]

75. Tatsumi T, Kierstead LS, Ranieri E, Gesualdo L, Schena FP, Finke JH, et al. Disease-associated bias in T helper type $1(\mathrm{Th} 1) / \mathrm{Th} 2 \mathrm{CD} 4(+) \mathrm{T}$ cell responses against MAGE-6 in HLADRB10401(+) patients with renal cell carcinoma or melanoma. J Exp Med. 2002; 196:619-628. [PubMed: 12208877]

76. Nishimura T, Nakui M, Sato M, Iwakabe K, Kitamura H, Sekimoto M, et al. The critical role of Th1-dominant immunity in tumor immunology. Cancer Chemother Pharmacol. 2000; 46(Suppl):S52-S61. [PubMed: 10950149]

77. Carballido JA, Molto LM, Manzano L, Olivier C, Salmeron OJ, Alvarez de Mon M. Interferonalpha-2b enhances the natural killer activity of patients with transitional cell carcinoma of the bladder. Cancer. 1993; 72:1743-1748. [PubMed: 8348503]

78. Brunet JF, Denizot F, Luciani MF, Roux-Dosseto M, Suzan M, Mattei MG, et al. A new member of the immunoglobulin superfamily--CTLA-4. Nature. 1987; 328:267-270. [PubMed: 3496540]

79. Khattri R, Auger JA, Griffin MD, Sharpe AH, Bluestone JA. Lymphoproliferative disorder in CTLA-4 knockout mice is characterized by CD28-regulated activation of Th2 responses. J Immunol. 1999; 162:5784-5791. [PubMed: 10229811]

80. Tivol EA, Borriello F, Schweitzer AN, Lynch WP, Bluestone JA, Sharpe AH. Loss of CTLA-4 leads to massive lymphoproliferation and fatal multiorgan tissue destruction, revealing a critical negative regulatory role of CTLA-4. Immunity. 1995; 3:541-547. [PubMed: 7584144]

81. Waterhouse P, Penninger JM, Timms E, Wakeham A, Shahinian A, Lee KP, et al. Lymphoproliferative disorders with early lethality in mice deficient in Ctla-4. Science. 1995; 270:985-988. [PubMed: 7481803]

82. Lin H, Rathmell JC, Gray GS, Thompson CB, Leiden JM, Alegre ML. Cytotoxic T lymphocyte antigen 4 (CTLA4) blockade accelerates the acute rejection of cardiac allografts in CD28-deficient mice: CTLA4 can function independently of CD28. J Exp Med. 1998; 188:199-204. [PubMed: 9653096]

83. Krummel MF, Allison JP. CD28 and CTLA-4 have opposing effects on the response of T cells to stimulation. J Exp Med. 1995; 182:459-465. [PubMed: 7543139]

84. Hurwitz AA, Foster BA, Kwon ED, Truong T, Choi EM, Greenberg NM, et al. Combination immunotherapy of primary prostate cancer in a transgenic mouse model using CTLA-4 blockade. Cancer Res. 2000; 60:2444-2448. [PubMed: 10811122]

85. Kwon ED, Foster BA, Hurwitz AA, Madias C, Allison JP, Greenberg NM, et al. Elimination of residual metastatic prostate cancer after surgery and adjunctive cytotoxic T lymphocyte-associated antigen 4 (CTLA-4) blockade immunotherapy. Proc Natl Acad Sci U S A. 1999; 96:15074-15079. [PubMed: 10611340]

86. van Elsas A, Hurwitz AA, Allison JP. Combination immunotherapy of B16 melanoma using anticytotoxic T lymphocyte-associated antigen 4 (CTLA-4) and granulocyte/macrophage colonystimulating factor (GM-CSF)-producing vaccines induces rejection of subcutaneous and metastatic 
tumors accompanied by autoimmune depigmentation. J Exp Med. 1999; 190:355-366. [PubMed: 10430624]

87. Hanson DCCP, Primiano MJ, Donovan CB, Gardner JP, Natoli EJ, Morgan RW, et al. Preclinical in vitro characterization of anti-CTLA4 therapeutic antibody CP-675,206. PROC AM ASSOC CANCER RES. 2004; 45 Abs 3802.

88. Ribas ADT, Comin-Anduix B, de la Rocha P, Glaspy JA, Economou JS, Gomez-Navarro J, et al. Changes in intratumoral immune cell infiltrates, Foxp3 and indoleamine 2, 3-dioxygenase (IDO) expression with the CTLA4 blocking MAB CP-675,206. J IMMUNOTHER. 2006; 29:636.

89. Urbani S, Amadei B, Tola D, Massari M, Schivazappa S, Missale G, et al. PD-1 expression in acute hepatitis $\mathrm{C}$ virus (HCV) infection is associated with HCV-specific CD8 exhaustion. J Virol. 2006; 80:11398-11403. [PubMed: 16956940]

90. Tarhini AA, Butterfield LH, Shuai Y, Gooding WE, Kalinski P, Kirkwood JM. Differing patterns of circulating regulatory $\mathrm{T}$ cells and myeloid-derived suppressor cells in metastatic melanoma patients receiving anti-CTLA4 antibody and interferon-alpha or TLR-9 agonist and GM-CSF with peptide vaccination. J Immunother. 2012; 35:702-710. [PubMed: 23090079]

91. Ferrantini M, Capone I, Belardelli F. Interferon-alpha and cancer: mechanisms of action and new perspectives of clinical use. Biochimie. 2007; 89:884-893. [PubMed: 17532550]

92. Curtsinger JM, Valenzuela JO, Agarwal P, Lins D, Mescher MF. Type I IFNs provide a third signal to CD8 T cells to stimulate clonal expansion and differentiation. J Immunol. 2005; 174:4465-4469. [PubMed: 15814665]

93. Gerner MY, Heltemes-Harris LM, Fife BT, Mescher MF. Cutting edge: IL-12 and type I IFN differentially program CD8 T cells for programmed death 1 re-expression levels and tumor control. J Immunol. 2013; 191:1011-1015. [PubMed: 23804712]

94. Fourcade J, Kudela P, Sun Z, Shen H, Land SR, Lenzner D, et al. PD-1 is a regulator of NYESO-1-specific CD8+ T cell expansion in melanoma patients. J Immunol. 2009; 182:5240-5249. [PubMed: 19380770]

95. Muhlbauer M, Fleck M, Schutz C, Weiss T, Froh M, Blank C, et al. PD-L1 is induced in hepatocytes by viral infection and by interferon-alpha and -gamma and mediates $\mathrm{T}$ cell apoptosis. J Hepatol. 2006; 45:520-528. [PubMed: 16876901] 